
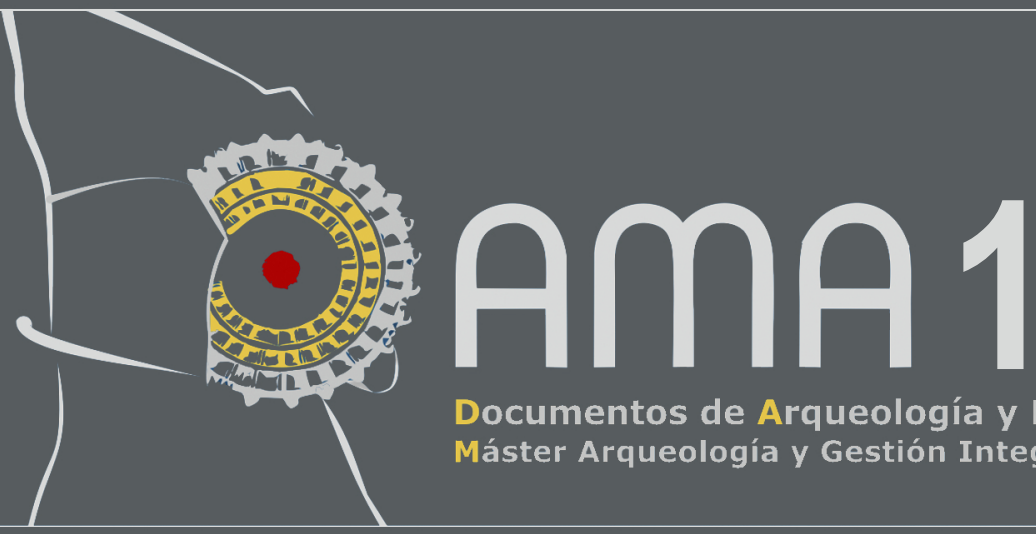

Documentos de Arqueología y Patrimonio Histórico

Máster Arqueología y Gestión Integral del Patrimonio de la Universidad de Alicante



Documentos de Arqueología y Patrimonio Histórico Revista del Máster Universitario en Arqueología Profesional y Gestión integral del Patrimonio 



\section{DAMA 1}

\section{6}

DOCUMENTOS DE ARQUEOLOGÍA Y PATRIMONIO HISTÓRICO DEL MÁSTER UNIVERSITARIO EN ARQUEOLOGÍA PROFESIONAL Y GESTIÓN INTEGRAL DEL PATRIMONIO DE LA UNIVERSIDAD DE ALICANTE 


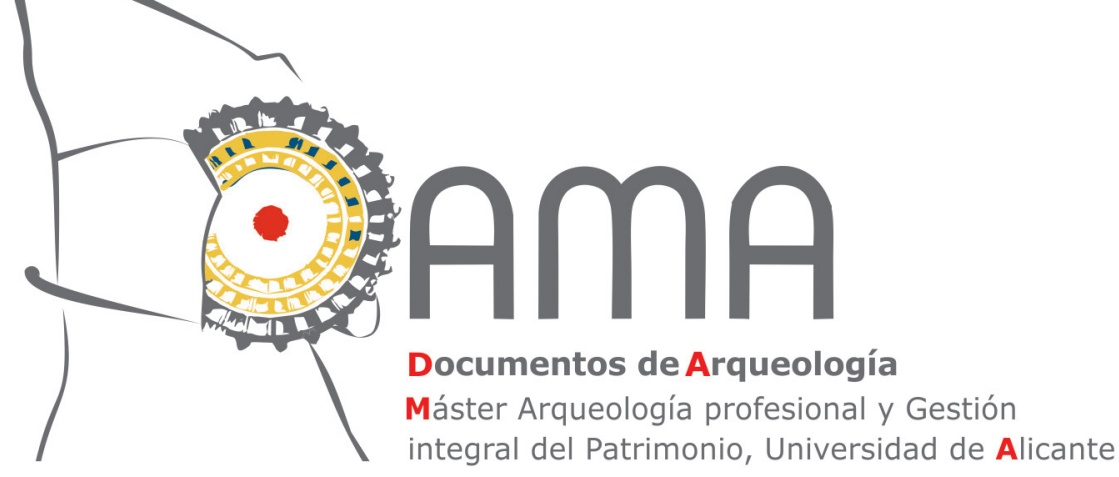

La revista electrónica DAMA. Documentos de Arqueología y Patrimonio Histórico surge como servicio para todos aquellos alumnos del Máster en Arqueología Profesional y Gestión Integral del Patrimonio de la Universidad de Alicante que se están iniciando en la investigación y cuya primera aportación a nuestra disciplina suele ser su Trabajo de Fin de Máster (TFM). Estos proyectos en muchos casos representan casi todo un curso de trabajo y esfuerzo, y con frecuencia quedan inéditos.

El objetivo de esta revista es ofrecer un medio que facilite la publicación de los resultados de sus TFM. La edición se presenta en versión digital y cuenta con su correspondiente ISSN. Se publica de forma anual en el sitio web de la Universidad de Alicante (http://web.ua.es/es/dama/) y en su repositorio (RUA). Los artículos publicados son descargables en formato PDF.

\section{Consejo de Redacción}

Directores (Coordinadores del Máster)

Carolina Doménech Belda

Fernando Prados Martínez

\section{Secretaria de Edición}

Julia Sarabia Bautista

\section{Vocales}

Los miembros de la Comisión Académica del Máster Universitario en Arqueología Profesional y Gestión del Patrimonio (http://dprha.ua.es/es/magip/comision-academica.html)

\section{Edita}

Máster Universitario en Arqueología Profesional y Gestión Integral del Patrimonio Departamento de Prehistoria, Arqueología, Historia Antigua, Filología Griega y Filología Latina

Facultad de Filosofía y Letras II

Universidad de Alicante

Ctra San Vicente del Raspeig s/n

E-03690 San Vicente del Raspeig (Alicante)

Web:http://dprha.ua.es

Teléfono: (+34) 965903663

Fax: (+34) 965903823

E-mail: revista.dama@ua.es

\section{ISSN}

$2530-2345$

\section{Portada}

Modelo fotogramétrico de un hypocaustum romano realizado por Ana Charquero 
EDITORIAL

Coordinación del máster

ENTREVISTA A SOLVEIG NORDSTRÖM: UNA MIRADA HACIA EL PASADO DE LA ESCUERA

Raúl Berenguer González ..

\section{ARQUEOLOGÍA Y MÉTODO}

EL ESTUDIO DE LOS MATERIALES CONSTRUCTIVOS DE TIERRA DEL CABEZO DEL POLOVAR (VILLENA, ALICANTE): APORTACIÓN A LAS FORMAS CONSTRUCTIVAS DE DOS PEQUEÑAS EDIFICACIONES CAMPESINAS DE LA EDAD DEL BRONCE EN EL LEVANTE PENINSULAR

María Pastor Quiles

VIVIENDAS POSTALAYÓTICAS: UNA APROXIMACIÓN A LOS ESPACIOS DOMÉSTICOS EN EL ARCHIPIÉLAGO BALEAR (550-123 ANE)

Octavio Torres Gomáriz

LAS FLOTAS DE GUARNICIÓN ROMANA EN LAS COSTAS DE HISPANIA A PARTIR DE LAS FUENTES EPIGRÁFICAS

Sergio Lledó Ramírez

UNA APROXIMACIÓN A LA RECONSTRUCCIÓN DEL PAISAJE HISTÓRICO: EL PROYECTO L'ALMISSERÀ Diana López Arroyo

NUEVAS PERSPECTIVAS PARA EL ESTUDIO ARQUEOLÓGICO DEL POBLAMIENTO RURAL MEDIEVAL EN ASPE (ALICANTE): HUERTAS Y ALQUERÍAS JUNTO AL RÍO TARAFA

Felipe Mejías López

ARQUEOLOGÍA DE LAS GUERRAS CARLISTAS

Iván Roldán Vergarachea

LA CERÁMICA DE USO ARQUITECTÓNICO EN NOVELDA: LA AZULEJERÍA DE FINALES DEL SIGLO XIX Y PRINCIPIOS DEL XX

Natalia Sala Pérez

PRÁCTICA Y USOS DE LA FOTOGRAMETRÍA DIGITAL EN ARQUEOLOGÍA

Ana $M^{a}$ Charquero Ballester.

\section{GESTIÓN Y PUESTA EN VALOR DEL PATRIMONIO}

ARQUEÓLOGOS CON DISCAPACIDAD. ARQUEOLOGÍA INCLUSIVA

Ana Samaniego Espinosa

"LES COVES DELS PESCADORS DE EL CAMPELLO": PROPUESTA DE RECUPERACIÓN, PUESTA EN VALOR Y USO PÚBLICO DE UN PATRIMONIO OLVIDADO

Ana Isabel Castro Carbonell .

LA COLONIA DE SANTA EULALIA. ESTUDIO Y PROPUESTA DE RECUPERACIÓN

Héctor de Arriba González

PROYECTO DE PUESTA EN VALOR DE LOS RESTOS DE LA GUERRA CIVIL EN LA CIUDAD DE ALICANTE

Leticia Victoria González Chouciño

ANTEPROYECTO MUSEOGRÁFICO DEL MUSEO DE HISTORIA DE SAX

Alberto Ochoa García

EL CONGRESO DE MÁSTER: UNA PROPUESTA DIDÁCTICA DEL MÁSTER DE ARQUEOLOGÍA DE LA UNIVERSIDAD DE ALICANTE

Ignasi Grau Mira, Sonia Gutiérrez Lloret, Carolina Doménech Belda, Julia Sarabia Bautista 



\title{
LA CERÁMICA DE USO ARQUITECTÓNICO EN NOVELDA: LA AZULEJERÍA DE FINALES DEL SIGLO XIX Y PRINCIPIOS DEL XX
}

\author{
Natalia Sala Pérez
}

\section{RESUMEN}

El objetivo de este trabajo es analizar y dar a conocer el uso de la azulejería en la arquitectura de Novelda (Alicante), tanto como elemento decorativo como funcional, enmarcado en un período cronológico que abarca desde finales del siglo XIX hasta principios del XX. Se trata de un patrimonio muchas veces poco valorado y mal protegido que es necesario poner en valor.

Palabras clave: azulejería, cerámica, arquitectura, siglos XIX-XX, Novelda

\section{ABSTRACT}

The objective of this project is to analyze and to make public the use of tiling in Novelda's splendid architecture, as well as the functional and decorative elements, chronologically dating back to the end of the XIX century to the beginning of the XX century. This heritage is of important historical value and must be protected and recognised as such.

Key Words: tiling, pottery, architecture, XIX-XX century, Novelda. 


\section{INTRODUCCIÓN}

La ciudad de Novelda, ubicada en el valle del Vinalopó en la provincia de Alicante, no ha tenido nunca una industria azulejera, sin embargo ha seguido siempre las modas del momento, y muestra de ello son los elementos que hemos estudiado en este trabajo: los aleros decorados con azulejos, las placas identificativas de calles y de numeración de inmuebles y el revestimiento de paredes.

La elección de este tema se debe principalmente a que la azulejería es un tipo de patrimonio cuyo valor no está totalmente reconocido por la sociedad ${ }^{1}$, a pesar de que estos elementos son una muestra de nuestro pasado más reciente. En el caso concreto de los azulejos que se encuentran en los aleros de las viviendas, a la altura a la que se encuentran y su posición hacen que muchas veces pasen desapercibidos. Por todo ello, lo que pretendemos con este trabajo es aproximarnos a la historia de esta ciudad, sobre todo a un período de tan importantes cambios; así como también documentar y poner en valor un elemento material que es parte del Patrimonio Histórico y que se está perdiendo.

Con esto, los objetivos que nos hemos propuesto son los siguientes:

1) Documentar, inventariar y catalogar los elementos de azulejería conservados en el casco urbano de Novelda y que se engloban en este período cronológico, así como también conocer el grado de implantación de su uso.

2) Reconocer las diferentes áreas urbanas a través de dicho uso determinando las zonas de fuerte implantación de las familias burguesas, que serán quienes más uso hagan de este tipo de elemento.

3) Determinar la procedencia y origen de los azulejos a partir de los motivos decorativos, así como también establecer una cronología lo más aproximada posible, tanto de la decoración como de los edificios a ellos asociados.

4) Detectar los azulejos de serie de uso frecuente frente a tipos únicos o realizados por encargo, que serán la minoría, y establecer diferencias entre ambos casos.

\section{LA TRADICIÓN AZULEJERA}

La función principal de los azulejos, en su origen, era aumentar las medidas higiénicas en una sociedad que sufría una gran cantidad de infecciones. Para evitar esto se revestían los baños y las cocinas con azulejos, pero esta moda fue cambiando con el tiempo y su uso se extendió a varios ámbitos, con un papel fundamental como elemento decorativo.

Los avances de la industrialización permitían reducir costes y mejorar su calidad, lo que suponía a su vez un aumento de su consumo y de su popularización. Además, gracias a este desarrollo industrial, las técnicas decorativas también cambiaron, pasando de las más costosas por el tiempo empleado (estarcido) a otras mucho más rápidas (sistema de trepas y cuerda seca). De esta manera se pasará de un tipo de producción artesanal a otro industrial. En España, la etapa fundamental en la cual se van a producir este cambio en el tipo de producción va a ser la que tiene lugar entre la Década Moderada (1844) y la Exposición Universal de Barcelona (1888). La producción azulejera se va a concentrar en cuatro núcleos: Sevilla, Barcelona, Valencia y Onda (Estall y Poles, 2000, pp. 20-21).

Durante el siglo XIX, que es el período en el que nos vamos a centrar, Valencia y Onda van a ser los grandes productores azulejeros del momento más próximos geográficamente a la población que estudiamos. Hasta la primera mitad del siglo la industria azulejera se concentraba en la ciudad de Valencia, pero a partir de la segunda mitad empezarán a aparecer nuevas industrias azulejeras en otros puntos geográficos que se van a localizar mayoritariamente en la provincia de Castellón donde destacará la ciudad de Onda y donde se van a instalar muchos de los productores azulejeros

1 Así lo han puesto de manifiesto autores como I. Pérez Guillén y J. Feliu Franch. 
valencianos. Onda va a ser el gran centro productor hasta la actualidad, aunque habrá otros centros de menor importancia (Pérez Guillén, 2000; Estall y Poles, 2000).

Novelda no tuvo nunca una industria azulejera propia, por ello, la alta burguesía de la población, siguiendo las modas del momento, adquirió azulejos para las lujosas viviendas que se estaban construyendo. Su uso no se limitó a los espacios interiores de estas residencias, sino que fueron utilizados también en los aleros exteriores de los tejados ${ }^{2}$ como muestra de su estatus económico. En muchos casos no es posible determinar el centro productor de estos azulejos ya que no podemos observar los reversos (espacio donde las fábricas colocaban su firma) puesto que se encuentran colocados en su lugar original.

\section{LA EXPANSIÓN URBANA DE NOVELDA}

Entre finales del siglo XIX y principios del XX Novelda empieza a experimentar una serie de cambios muy importantes debido al crecimiento económico y demográfico, que dará lugar a un crecimiento urbanístico, con nuevas calles, nuevas plazas, nuevas viviendas.... Junto al aumento del cultivo de la vid y la producción de vino y aguardiente, se desarrolló una industria del mármol, así como la comercialización del azafrán y otros productos manufacturados. Con estos cambios se pasó de una economía agrícola a una más dinámica y diversificad, factores que propiciaron el auge de una dinámica clase burguesa.

A partir de la información aportada por J.J. Martínez Martínez (2001) sabemos que hasta 1884 Novelda estaba formada por dos núcleos: la Novelda propiamente dicha, que sería el núcleo más antiguo, y el barrio de San Roque, zonas que estaban unidas por la calle de San Roque y entre las cuales quedaba un amplio espacio ocupado por campos de cultivo. Pero a finales de siglo se produce un crecimiento demográfico y económico muy importante que da lugar a la necesidad de ampliar la superficie de la villa puesto que la ciudad estaba saturada, siendo necesaria la construcción de nuevas viviendas para alojar a la población que iba en alza.

Si antes el espacio se ampliaba construyendo nuevas casas en los márgenes de los caminos, es decir, no había un plan en la ordenación urbana, ahora parecía lógico que se utilizara ese espacio vacío existente entre el núcleo principal de Novelda y San Roque, tierras que pertenecían a la Duquesa de Medina Sidonia, quien las cedió al Ayuntamiento para la ampliación de la villa. (Fig.1)

Dicha zona de ampliación concentrará un gran número de viviendas residenciales burguesas que presentan vistosos ejemplos de aleros decorados con azulejaría. Lo mismo ocurre con la calle que se abrió en 1905 (Maestro Segura, casas $14,15,16,17)$ para comunicar el nuevo barrio de medina-Sidonia con la zona antigua, y que va a resultar de un gran atractivo para las clases burguesas imperantes, que a inicios del

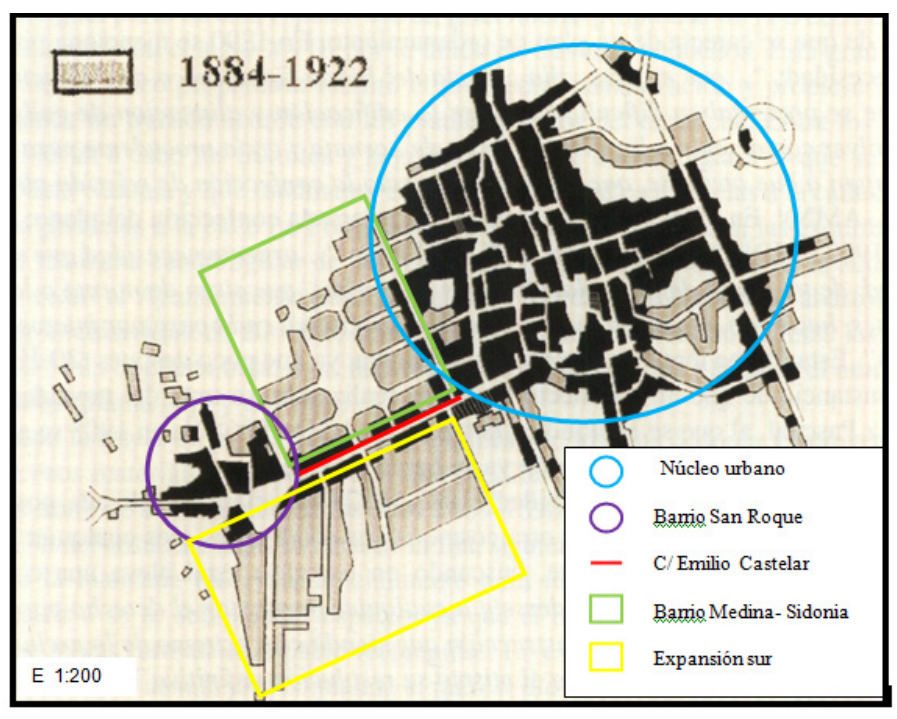

Fig. 1. Plano de la expansión urbana de Novelda entre 1884-1922. Elaboración de la autora a partir del original de J.J Martínez. (2001). siglo XX construirán sus viviendas donde aún se conservan buenos ejemplos de azulejería (Fig. 2).

2 Otro uso habitual del uso de la azulejería eran los reversos de los balcones, pero en el caso de Novelda no hemos encontrado ningún ejemplo que podamos incluir en el período histórico que hemos estudiado. 


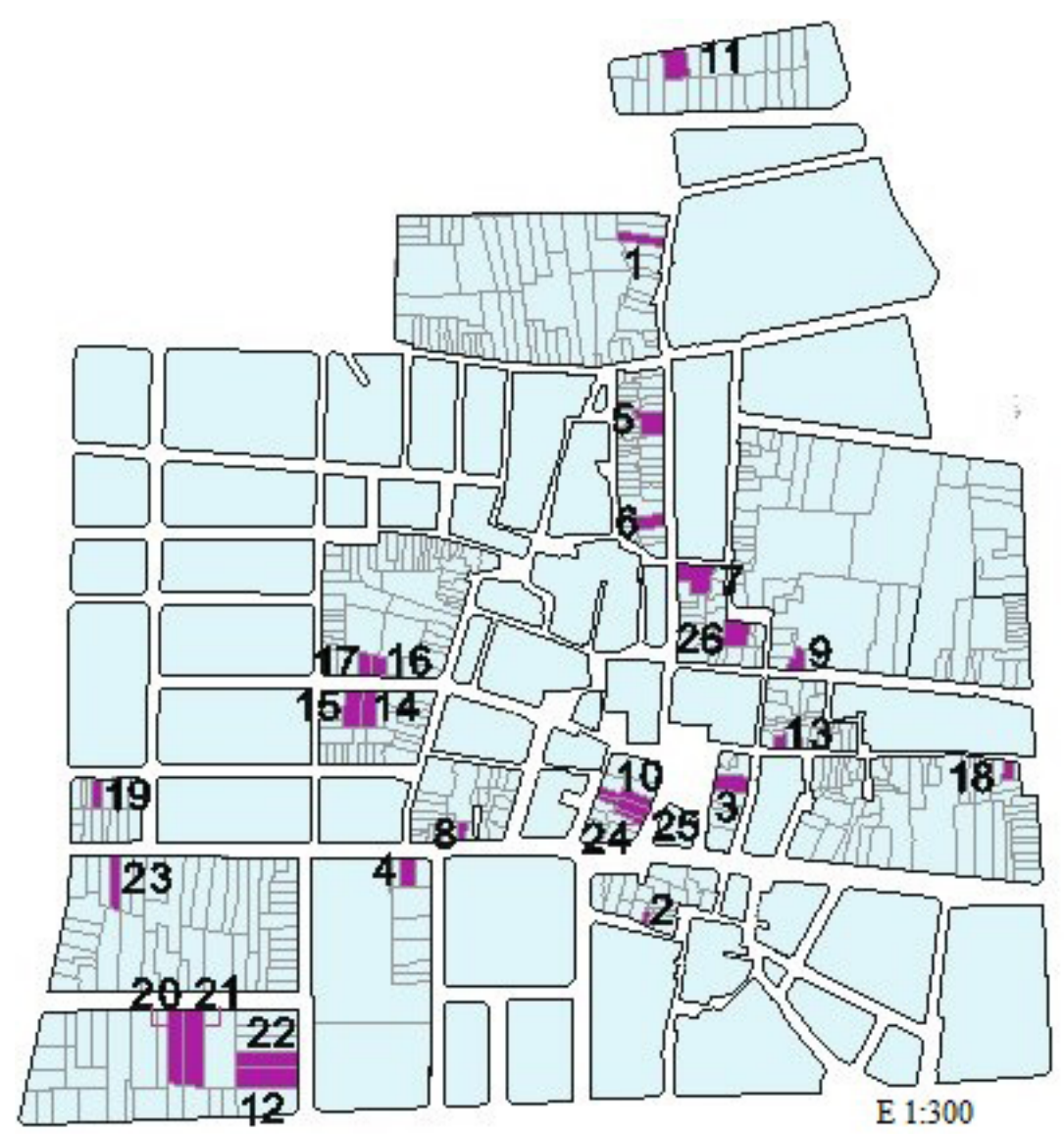

Fig. 2. Plano zona prospectada que señaliza los inmuebles con aleros cerámicos. Elaboración propia.

Por lo que respecta a la expansión urbana hacia el sur de la calle de San Roque, se caracteriza principalmente por un trazado irregular, sin simetría e independiente. En general se trataba de un barrio de gente con renta más baja, sin embargo cabe destacar la C/ Jaume II y C/ Argentina (12, 20, 21,22 ) que, por su proximidad al Casino, fue elegida también por algunas familias burguesas para construir sus viviendas, las cuales aparecen decoradas con aleros de azulejos.

Pero, como podemos ver en el plano anterior, la mayoría de inmuebles con aleros de azulejos las encontramos en la zona más antigua, en el casco histórico.

Durante el siglo XIX se va a ir desarrollando esa burguesía que va a seguir, en principio, la corriente academicista, lo que se plasmará en sus viviendas de la siguiente manera: arquitectura simétrica, con rejerías forjadas, un revestimiento vivo de la fachada. A partir de la segunda mitad de siglo ese proceso de enriquecimiento unido a la transformación de la imagen de la ciudad se va a intensificar. Pero será entre finales del siglo XIX, y sobre todo a principios del siglo XX, cuando estas familias burguesas van a hacer más patente esa ostentación pública de su poder económico con la reedificación de sus viviendas que, siguiendo el esquema academicista anteriormente explicado, incorporarán nuevos elementos como los aleros de cerámica (algunos de ellos siguiendo la estética modernista), ménsulas, etc. Y relacionado con esto cabe recordar que Novelda pasó de villa a ciudad en el año $1901^{3}$, hecho que favoreció la pujanza económica de la ahora ciudad.

Junto a este tipo de viviendas academicistas surgirán también otras que seguirán las formas y características del estilo modernista, y que serán el máximo exponente del enriquecimiento de esa burguesía imperante del momento. Entre estos edificios encontramos los siguientes: la actual

3 Real Decreto del 3 de octubre de 1901 firmado por la Regente María Cristina. 
“Casa- Museo Modernista” y el actual “Centro Cultural Gómez- Tortosa”, el "Santuario de Santa María Magdalena" y la vivienda particular conocida como "Casa Mira”. Todo este conjunto se va a caracterizar por un refinamiento de la estética y decoración, cuya monumentalidad es reconocida incluso en la actualidad.

\section{LOS PRIMEROS ALEROS DECORADOS}

Según la información aportada por J. Coll Conesa (2000), tradicionalmente las casas populares no presentaban decoración en la fachada a excepción de las familias más pudientes, quienes utilizaban esta técnica para demostrar su poder económico con respecto al resto de la población. Algunos de los lugares privilegiados elegidos eran los patios, los porches, pero sobre todo los aleros, elementos estos últimos que son objeto de estudio de nuestro trabajo, y que se podían decorar siguiendo diversas técnicas como por ejemplo incorporando madera, piedra e incluso pintura.

Los aleros se construían originalmente con fines utilitarios para proteger las fachadas, (a pesar de que con el tiempo la única función de los aleros será la decorativa). Pero necesitaban estar hechos con materiales resistentes a las injerencias climatológicas, y por eso se pasó de utilizar cañizos y madera para su construcción a utilizar "rajoles"4.

En el caso de Novelda son numerosas las viviendas que tienen este tipo de "rajoles" en sus aleros pero, sin duda, destaca una que conserva un alero muy peculiar compuesto por dos hiladas de 34 ladrillos, todos pintados con motivos arquitectónicos, epigráficos, numéricos y simbólicos a excepción de 6. El motivo más representado es lo que se ha interpretado como la silueta de una cúpula, sin embargo lo que más llama la atención es la indicación de una fecha: "año 1860", que posiblemente fue el año en el que se construyó la vivienda (Fig.3).

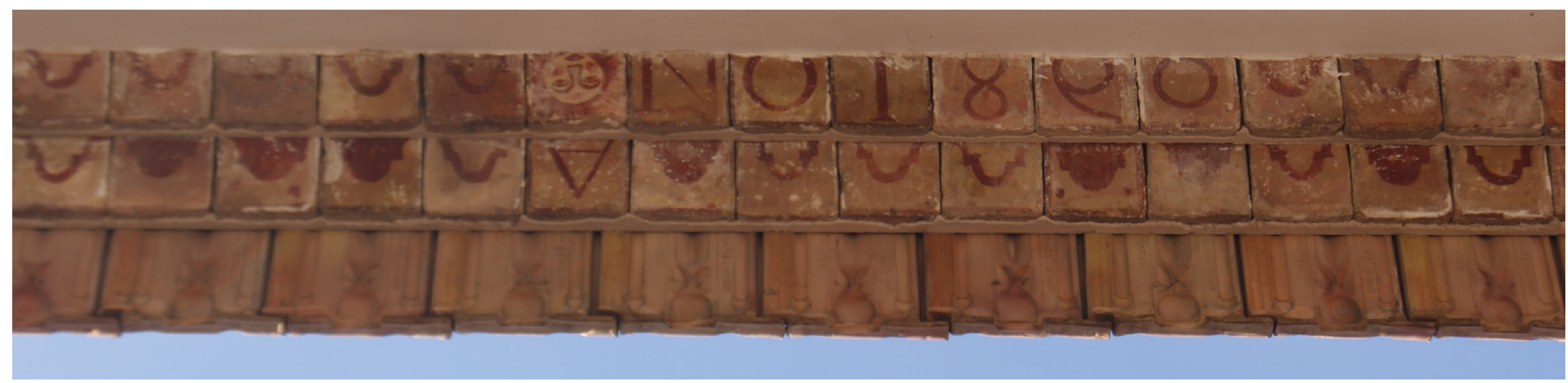

Fig. 3. Alero con ladrillos pintados en C/ San Rafael nº 15. Fotografía de la autora

Este tipo de elementos han sido interpretado por C. Navarro Poveda (2002) como manifestaciones de la arquitectura popular y un modo de indicar un estatus social superior por parte del propietario del edificio, su profesión o la actividad a la que estaba dedicada la casa. Según sus palabras se podría considerar "como elementos arquitectónicos precedentes a los bonitos y delicados aleros realizados en losetas cerámicas tan característicos de la arquitectura modernista y que tan bellos y variados ejemplos tenemos en los edificios construidos en Novelda por la burguesía local de principios del siglo XX" (Navarro Poveda, 2002, p. 94).

4 Se denominan así a las piezas de barro cocido, figurando así en los documentos desde la Edad Media. 


\section{LOS ALEROS DECORADOS CON AZULEJOS}

En Novelda, a partir de finales del siglo XIX y principios del XX, esa nueva clase social que surge como consecuencia del desarrollo económico que se estaba produciendo, la burguesía, inicia una tradición popular basada en la modificación de las viviendas con la incorporación de nuevos elementos arquitectónicos entre los que destacamos los aleros de gran vuelo decorados con azulejos.

De las 25 casas que hemos contabilizado con este tipo de aleros, hemos podido establecer una serie de conclusiones y rasgos en común.

\subsection{Características generales}

Se trata de azulejos de serie ${ }^{5}$, con unas medidas de 20 x $20 \mathrm{~cm}$ como consecuencia de la adaptación al Sistema Métrico Decimal. Estos azulejos pueden formar una composición bidireccional (el motivo decorativo continúa por dos de sus lados) o tetradireccional (el motivo decorativo continúa por sus cuatro lados).

\subsection{Cronología de los azulejos}

Este trabajo se centra en las últimas décadas del siglo XIX y principios del XX. Ajustar la cronología de los azulejos resulta más fácil si éste aparece en los catálogos existentes como son: "Catálogo de la colección de azulejos de serie del siglo XIX" y “Col•lecció de taulells Sergio León”. Sin embargo hay que tener en cuenta que la fecha aportada por estos catálogos es anterior a los azulejos documentados en Novelda. Esta circunstancia se puede explicar de dos maneras: o los azulejos se hicieron en la época indicada y se compraron estando hechos ya, o el motivo se eligió de un catálogo porque seguía estando de moda. Con todo esto, y a rasgos generales, nuestros azulejos los dataremos a principios del siglo XX.

\subsection{Lugar de producción}

Este punto nos resulta un tanto problemático y tenemos que tener en cuenta varios factores. En la bibliografía que hemos consultado se indica el lugar de producción e incluso la fábrica, pero tenemos que tener en cuenta que en esos casos se trata del lugar de fabricación de la pieza catalogada y no del modelo estudiado. En este sentido hay que tener en cuenta que un mismo modelo era copiado por diferentes fábricas de distintos lugares, por lo que la única manera de saber su lugar de producción exacto es observando las marcas del reverso, en el caso de que las llevara. Sin embargo esta tarea nos ha resultado imposible en nuestro caso por tratarse de azulejos que están todavía colocados en los aleros en su posición original que sólo permite observar los anversos.

\subsection{Técnica decorativa}

Con el desarrollo de la industrialización, las técnicas decorativas irán evolucionando. En este caso, nuestros azulejos se basan en dos técnicas distintas:

- Sistema de trepas: la trepa era un papel encerado que llevaba recortado el dibujo que debía rellenarse con un color. La combinación de varias trepas creaba la decoración final del azulejo y cuantos más colores llevara el diseño y más complicado fuera, más trepas necesitaba. Este tipo de decoración, por estar realizada con brochas pequeñas, era más rápida y daba unos resultados más homogéneos.

- Sistema de cuencas o arista: consiste en tallar en negativo el motivo del azulejo en un molde. A continuación, ese molde se presiona sobre la pieza bizcochada aún tierna sobre la que quedarán impresas unas pequeñas aristas que servirán más tarde para separar los diferentes colores de óxidos

5 En los azulejos de serie se repite siempre el motivo decorativo, aunque en algunos casos varíe el color. Por esta razón es común encontrar los mismos azulejos en varias viviendas e incluso en varias localidades. Este tipo de azulejos se diferencia de los de encargo en que estos últimos representan motivos decorativos elegidos por un particular. 
y esmaltes. Tras una primera cocción de estos azulejos se aplican los colores y se vuelven a cocer las piezas. En algunos casos, los motivos decorativos recibirán un retoque final a mano alzada.

\subsection{Motivos decorativos}

En cuanto a los motivos decorativos, los englobamos en dos grupos:

- Los modernistas: propios del momento histórico que está viviendo la cuidad en estos momentos, puesto que Novelda es un referente del Modernismo, formando parte incluso en la actualidad de la "Ruta Europea del Modernismo". En este grupo los motivos decorativos se basan en la representación de elementos de la naturaleza tanto de manera realista como abstracta (Fig. 4).
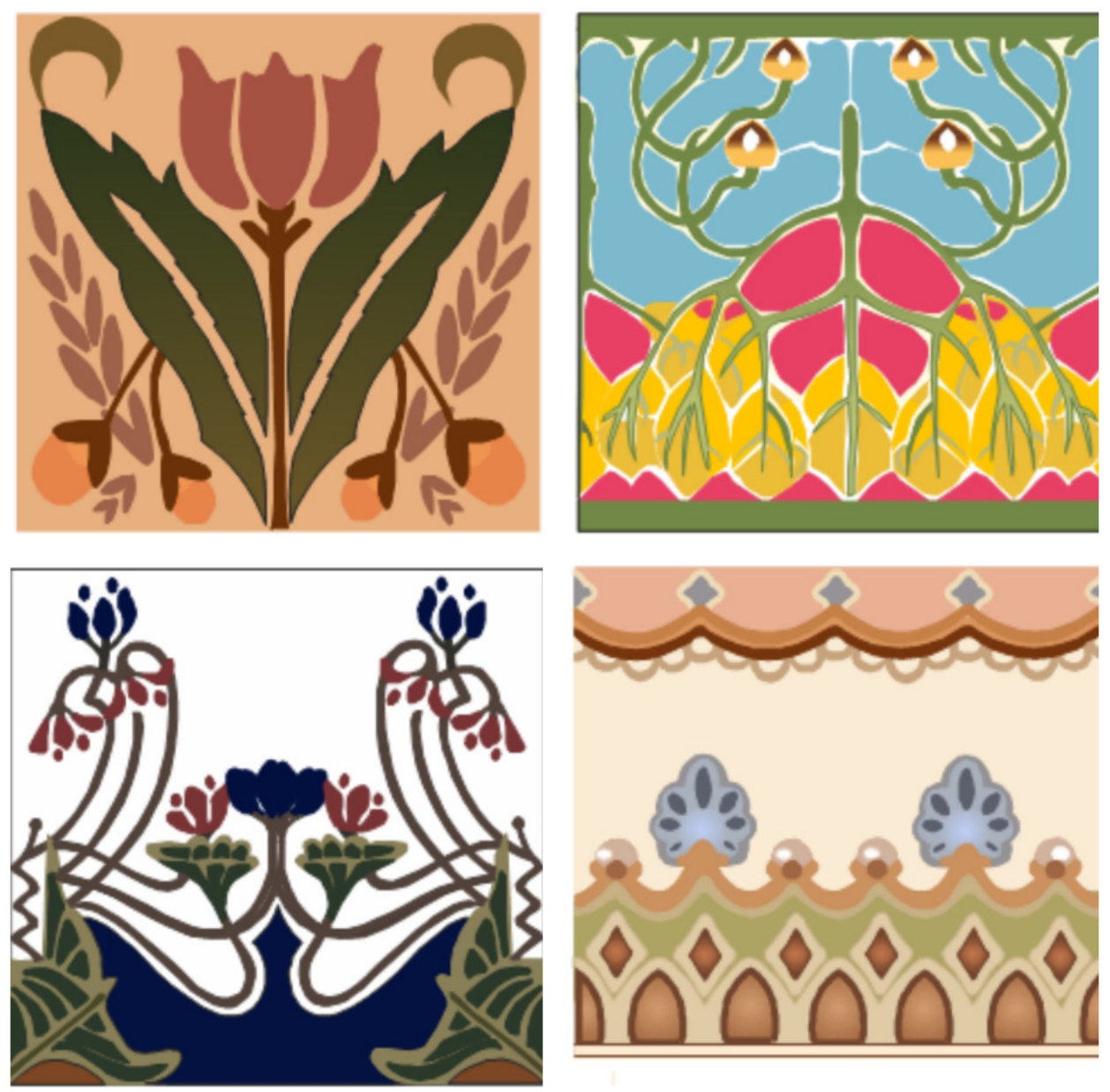

Fig. 4. Azulejos modernistas. Dibujos aproximados realizados por la autora.

- Los eclecticistas o "neo": este tipo de azulejos se basa en la utilización de motivos decorativos de épocas pasadas. De esta manera encontraremos los neogriego, neogótico, neo-renacimiento, neobarroco y neo-oriental (Fig. 5).

Dentro de los azulejos del conjunto modernista nos llama la atención la utilización de uno concreto que se repite en cinco inmuebles, además de en el interior de la Casa Museo Modernista. Se trata del azulejo de los claveles compuesto por un motivo central, un clavel blanco cuyo tallo y hojas están representados con líneas curvas a modo de cintas entrelazadas. Este conjunto está delimitado en su parte superior e inferior por una cenefa, también con la línea curva como protagonista. Estos azulejos presentan variaciones en cuanto a la combinación de sus formas, dando lugar así a composiciones distintas (Fig. 6). 

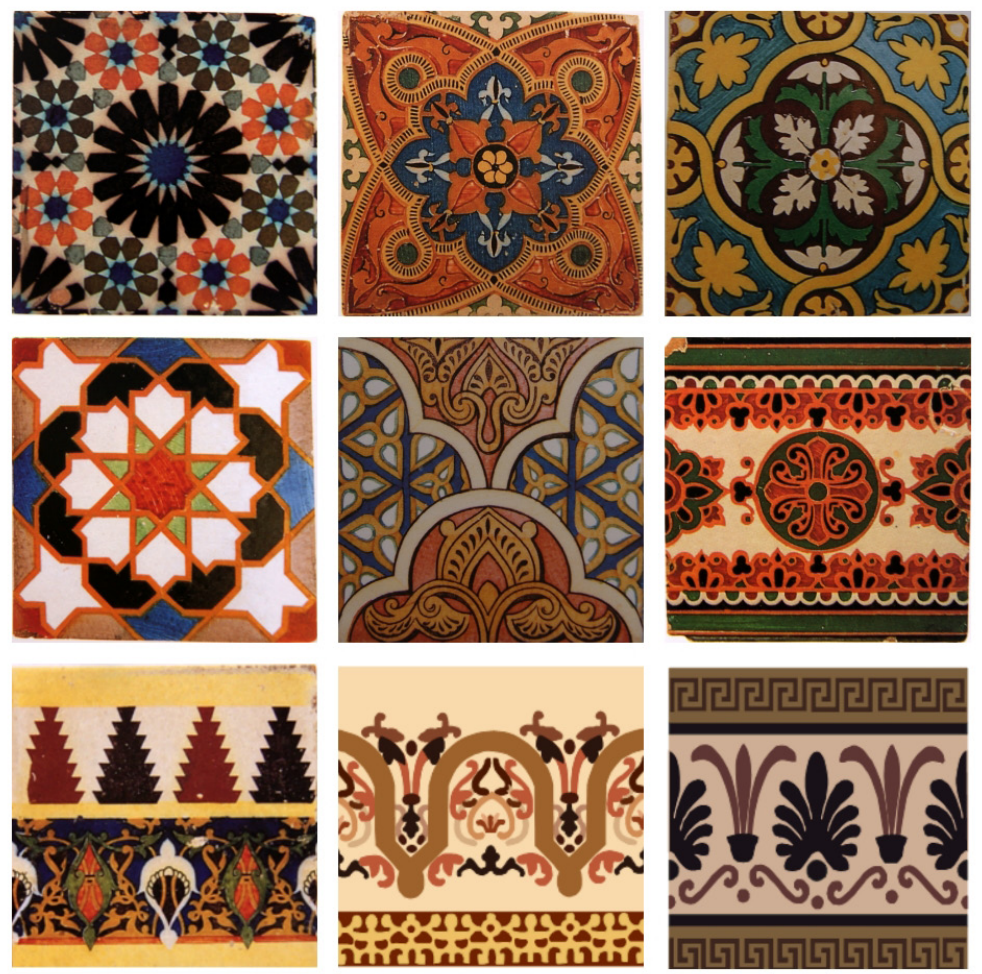

Fig. 5. Azulejos de estilo ecléctico. Motivos extraídos del "Catálogo de la colección de azulejos de serie del siglo XIX", a excepción de los dos últimos que son dibujos aproximados realizados por la autora.
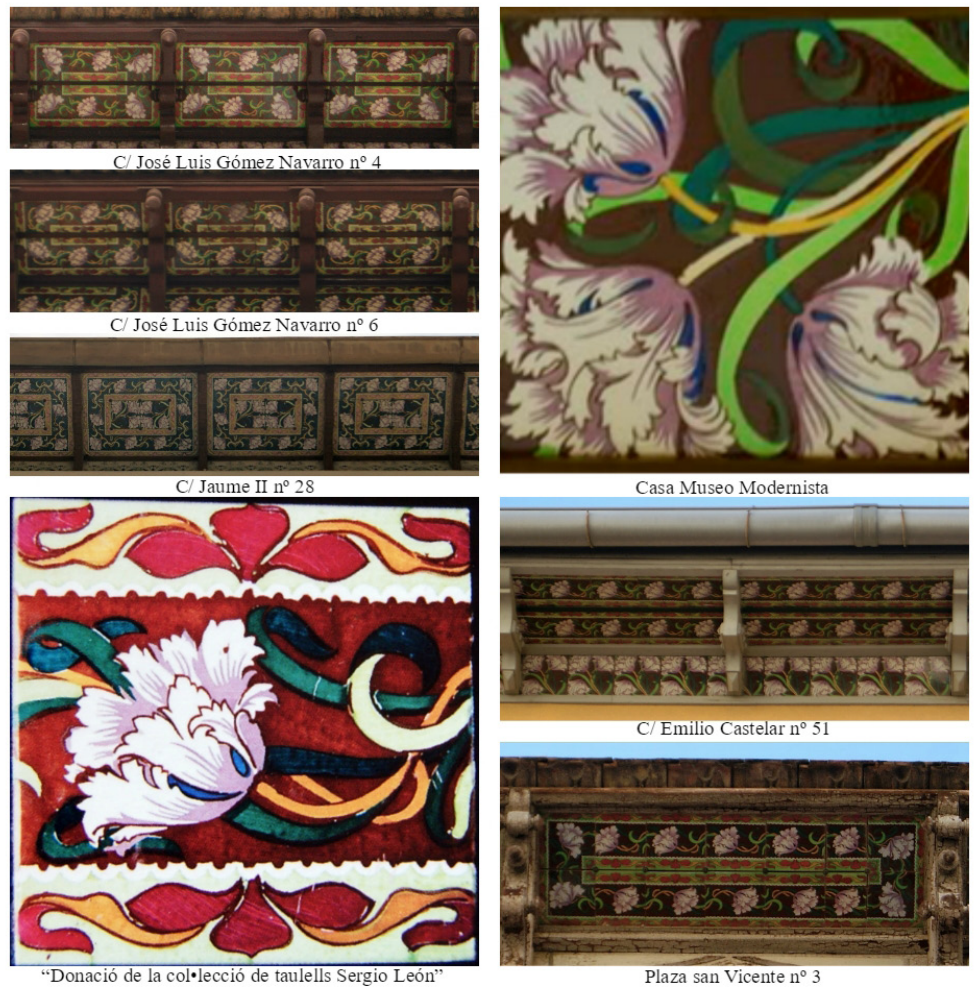

Fig. 6. Aleros y azulejos con el motivo de los claveles. Fotografía de la autora

\section{REVESTIMIENTO DE PAREDES}

La tradición de revestir las paredes de las casas se remonta a época medieval islámica, momento en el que se puso de moda los zócalos revestidos con azulejos, cuya tradición e incluso cuyos motivos decorativos se siguen utilizando en la actualidad. Ya en el siglo XVIII se revestían las paredes de las cocinas y de los cuartos de baño por cuestiones meramente higiénicas y de salubridad al ser lugares de posibles infecciones. Y con el tiempo este uso se convirtió en una moda, siendo habitual cubrir las paredes de cualquier estancia con azulejos por cuestiones meramente decorativas.

En este sentido podemos establecer una diferencia entre dos tipos de producciones:

1. Los azulejos de serie, que son la mayoría de los casos y que consiste en azulejos cuyos motivos se eligen de entre una gran cantidad de modelos distintos, ya elaborados de antemano o mediante un catálogo.

2. Los azulejos de encargo, que son los menos requeridos puesto que sólo las familias más pudientes podían encargar azulejos con los dibujos o modelos decorativos deseados, hechos exclusivamente para ellos.

Para la realización del estudio de este tipo de azulejos cabe destacar la complejidad que conlleva el hecho de que la mayoría de revestimientos de paredes son de estancias interiores y no de fachadas exteriores, por lo que nos ha sido imposible realizar un estudio exhaustivo. De esta manera nos hemos centrado únicamente en las dos viviendas modernistas abiertas al público actualmente (la Casa- Museo Modernista y el Centro Cultural Gómez- Tortosa).

Y para este artículo hemos decidido seleccionar los azulejos de encargo que se ubican en el patio de la Casa- Museo Modernista por su singularidad y belleza. 
Estos azulejos están pintados a mano y representan las distintas propiedades que pertenecían a Antonia Navarro Mira, así como también paisajes y actividades cotidianas. Antonia Navarro, la propietaria, fue quien encargó su elaboración por deseo propio, como muestra de su poder económico. Podemos contabilizar 23 motivos distintos, repetidos y con ligeras variaciones entre ellos puesto que están pintados a mano (Fig. 7).
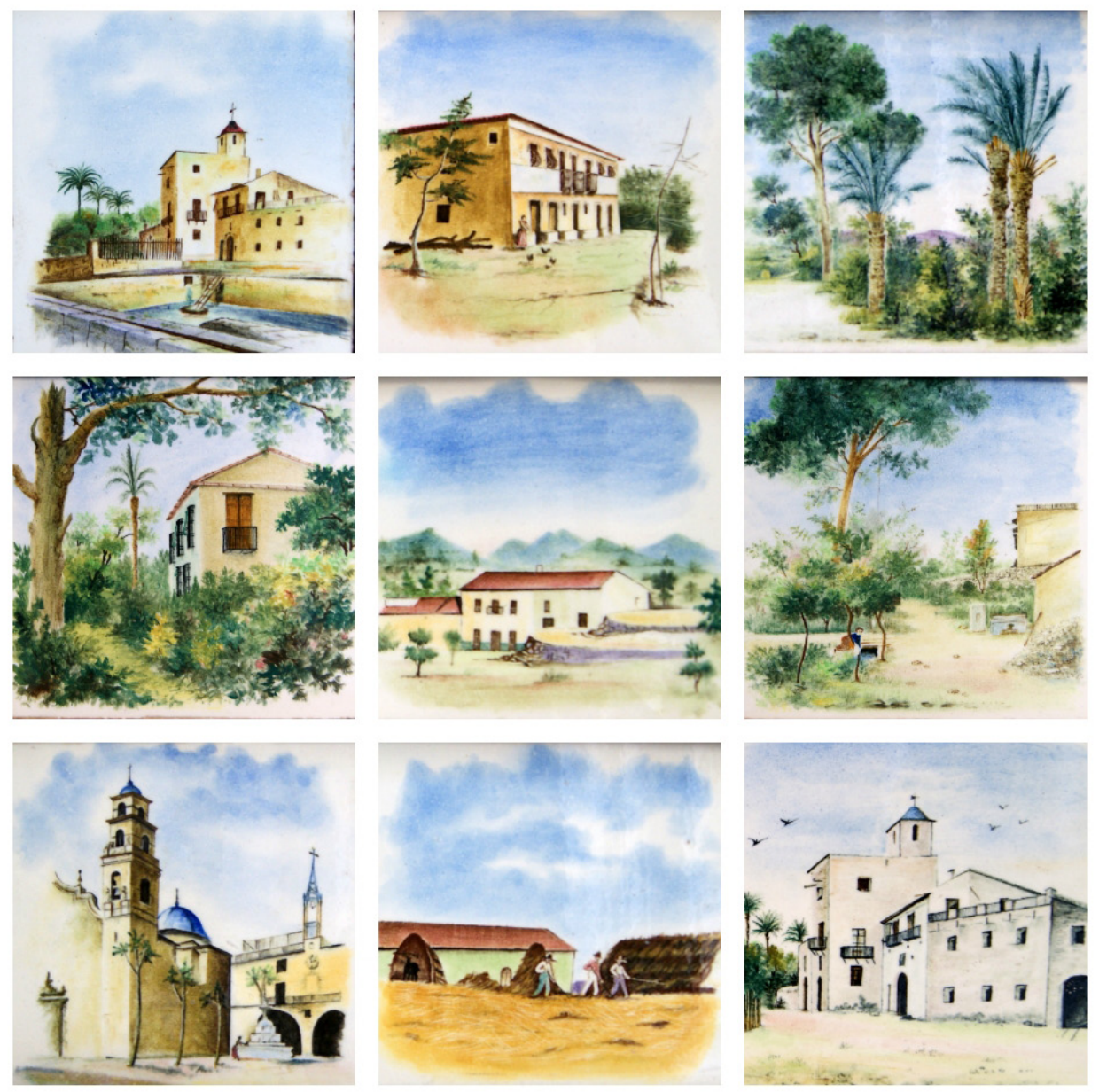

Fig. 7. Azulejos de encargo del patio de la Casa Museo Modernista. Fotografía de la autora.

\section{LAS PLACAS IDENTIFICATIVAS}

En este punto vamos a hablar fundamentalmente de dos tipos de placas distintas: las que hacen referencia a la identificación de las calles y las que hacen referencia a la numeración de las casas. Relacionadas formalmente con ellas, trataremos también dos casos singulares: una placa de identificación de una ermita y una inscripción conmemorativa del momento en el que Novelda fue nombrada cabeza del partido judicial, esta última de gran interés por sus implicaciones cronológicas.

La utilización de placas cerámicas para estos usos parece lógica si pensamos en la gran durabilidad de los azulejos, pues hay que tener en cuenta su enorme capacidad de resistencia para soportar las variaciones climáticas. De hecho, su uso ha perdurado y sigue estando muy extendido actualmente.

Las placas a las que nos referimos se encuentran localizadas en el casco histórico de Novelda, en la zona más céntrica de la ciudad, y son pocos los ejemplos que se conservan de los más antiguos. El formato de los dos tipos de placas es el mismo, variando las medidas: se trata de un azulejo blanco (cuadrado en el caso de los números o rectangular en el caso de los nombres) con las letras y los 
números en negro. El color del fondo es el obtenido a partir de la cubrición del azulejo con la capa de esmalte estannífero (Fig. 8).

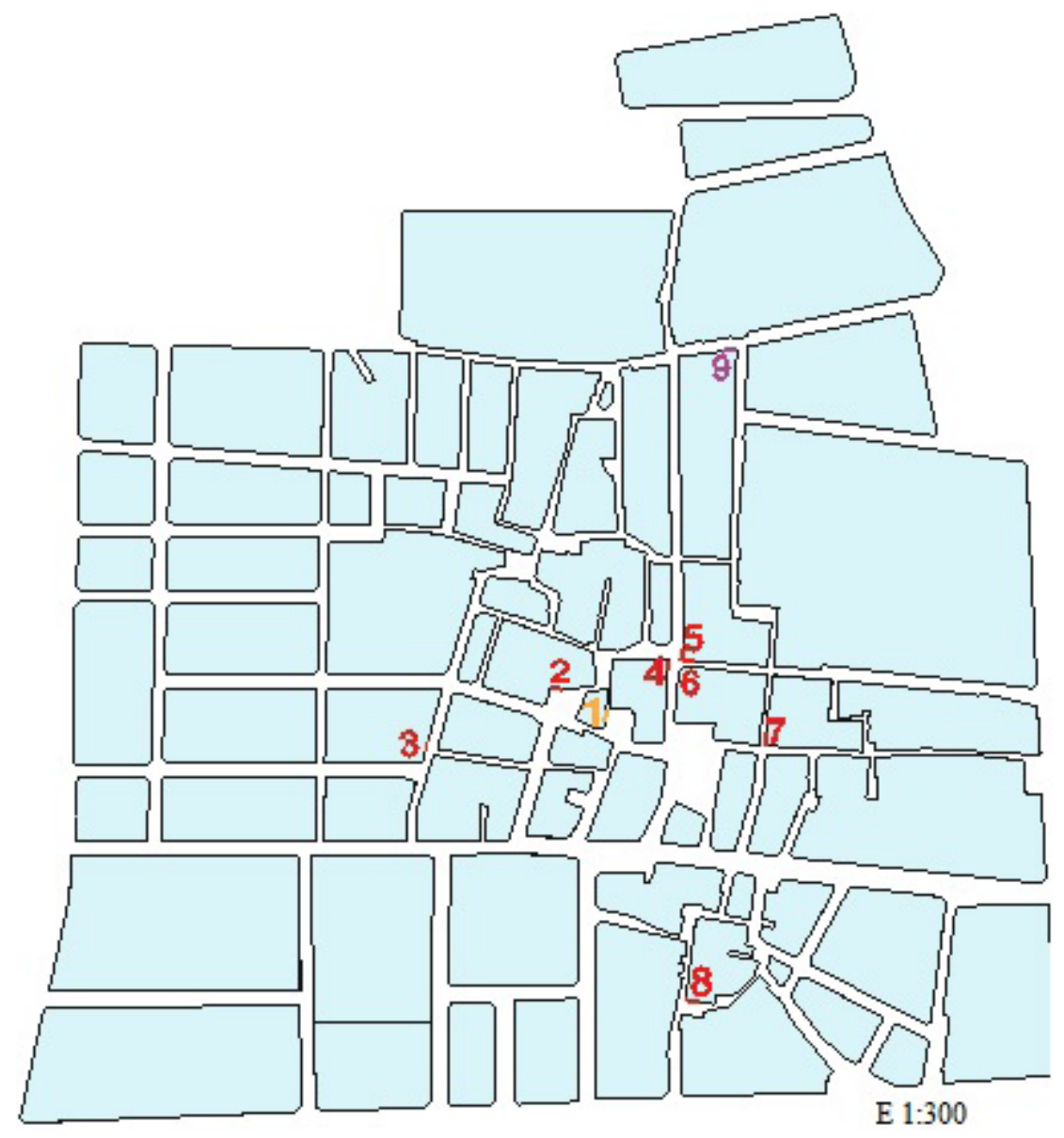

Fig. 8. Plano de la zona prospectada con la señalización de las placas identificativas. Elaboración propia.

Por lo que respecta a la cronología de la colocación de estas placas, nos han surgido problemas. En un principio nos decantamos por datar estas placas hacia los años 80 del siglo XIX, basándonos en un dato aportado por J. Feliu Franch $(1998,165)$ quien dice que a partir de 1880 se empezó a poner el nombre de las calles en placas de azulejos, a pesar de que su uso se remonta a época temprana. Sin embargo después de la lectura de publicaciones locales y tras la consulta de los cabildos municipales necesarios, hemos podido establecer nuestras propias conclusiones.

\subsection{Placas de identificación de calles}

Por lo que respecta a las placas conservadas de identificación de calles del XIX encontramos pocos ejemplos. Básicamente el formato es un azulejo blanco rectangular de $20 \times 30 \mathrm{~cm}$. con las letras negras (Fig.9).

No podemos decir hasta cuándo se siguieron utilizando estas placas, pero lo más probable es que fuera hacia el segundo tercio del siglo XX, momento en el cual este formato fue sustituido por otro distinto: las placas de identificación de calles pasarán a estar formadas por cuatro azulejos azules con las letras en blanco, siendo el formato más abundante puesto que se utilizó hasta fechas recientes cuando fue sustituido por las placas metálicas. 

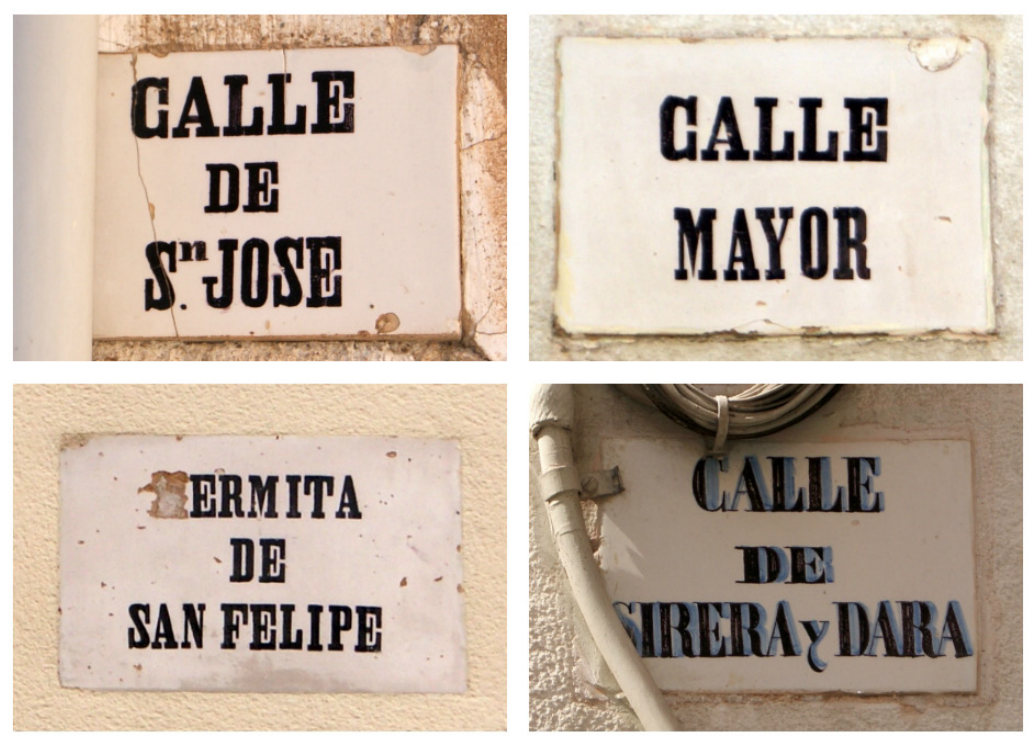

Fig. 9. Placas de identificación de calles. Fotografía de la autora. Fotografía de la autora.

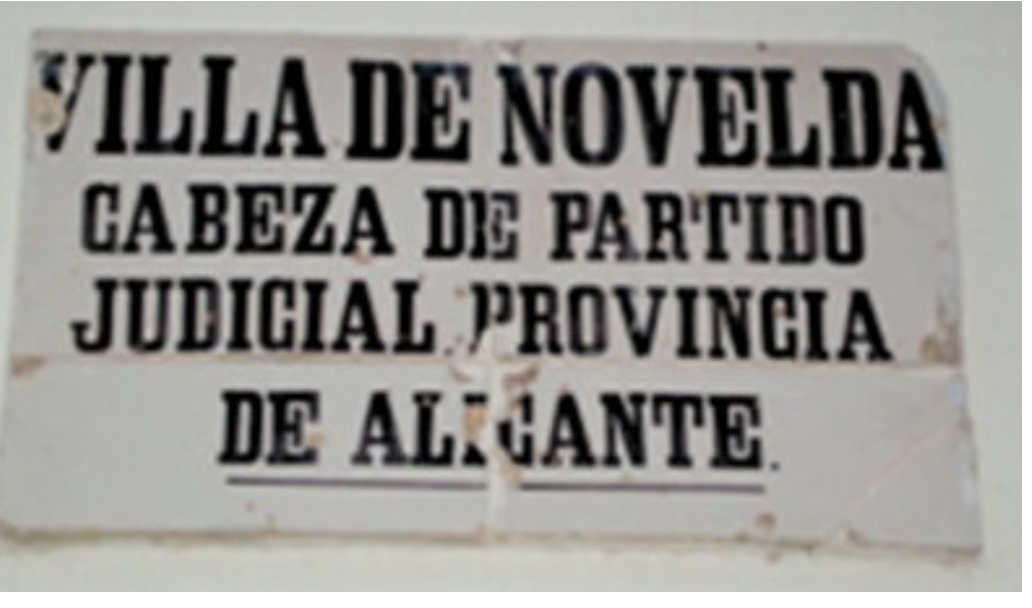

Fig. 10. Imagen original de la placa donde se aprecia su mal estado de conservación. Fotografía de la autora.

\subsection{Placas de numeración de inmuebles}

Estas placas presentan el mismo formato y características que las de identificación de calles (un azulejo blanco de 20 × $20 \mathrm{~cm}$. con los números en negro), por lo que serían coetáneas, localizándose también en el casco histórico de la ciudad. De este modelo se han conservado trece ejemplos, muchos de los cuales presentan un estado alterado por diversas razones: se han pintado, se han tapado con persianas, se han cubierto con cemento, se han perforado para la instalación eléctrica, etc.

Como ocurre en el caso de las placas de identificación de inmuebles, el formato cambia posteriormente siguiendo la estructura de las placas de identificación de calles (azulejo azul con números blancos).

\subsection{La cronología}

Existe una inscripción (Fig. 10) en la que se nombra a la Villa de Novelda como cabeza de partido judicial de la provincia de Alicante, integrando las poblaciones de Aspe, Monforte, Hondón de las Nieves, Hondón de los Frailes y Agost, hecho que tuvo lugar en el año 1836. En este sentido lo más lógico es pensar que esta placa se colocó ese mismo año en conmemoración de ese acontecimiento. Por tanto, como el resto de placas identificativas siguen el mismo formato, podemos deducir que 1836 es el año aproximado en el que se colocaron.

Además, gracias a la información aportada por F. Escolano Gómez (1959, e.p.) tenemos constancia de que en 1859 todas las calles cuyas placas hemos estudiado (a excepción de la Calle de Sirera y Dara de la cual se conserva la placa original aunque con caligrafía distinta al resto (Fig. 9)) tenían ese nombre. El mismo autor afirma que: "en el cabildo de 22 de abril de 1869, vino el amplio cambio de rotulación, sustituyendo viejos nombres por los de varios eminentes patricios que han trabajado a favor de la Libertad y como un debido recuerdo honorífico que debe pasar a la Historia, y que quedó acordado en los términos siguientes: (...) La de Salamanca... De Sirera y Dara (...)".

Esto se explica de manera que de todas las calles en las cuales hemos encontrado placas de identificación de calles, la última a la que se le cambió el nombre fue la de "Sirera y Dara", anteriormente llamada "de Salamanca". Y de esta manera el año 1869 podría ser en el cual se data esta placa. 


\section{CONCLUSIONES}

El objetivo principal de este trabajo era analizar y documentar los distintos usos de la azulejería en la arquitectura, averiguando su cronología y su lugar de producción. Para ello ha sido necesario realizar un exhaustivo trabajo de campo que ha permitido recopilar y documentar un importante número de elementos cerámicos conservados con distinto uso y función. Del estudio de dichos elementos se han podido extraer una serie de conclusiones.

La azulejería cerámica se ha utilizado tanto para uso decorativo como funcional. Entre los usos decorativos destacan los aleros de los tejados y los revestimientos de las paredes. Como uso funcional tenemos las placas de identificación de calles y de numeración de inmuebles. El mayor número de azulejos conservados corresponde a los decorativos, mientras que los identificativos son muy pocos los que han llegado hasta nuestros días.

En este sentido hemos averiguado que la mayoría de azulejos (los encontrados en los aleros de los tejados y recubriendo las paredes) se datan entre los últimos años del siglo XIX y los primeros del XX, mientras que las placas identificativas serían más antiguas de lo que pensábamos en un principio. A pesar de esto hemos mantenido el título original del trabajo puesto, en cualquier caso, esas placas identificativas eran las que estaban funcionando como tal a finales de siglo, estando datada la más reciente de las conservadas en 1869. Para la datación de las placas identificativas nos ha sido clave averiguar la fecha de cuando Novelda pasó a ser cabeza de partido judicial. Así, hemos podido tomar un año concreto como referencia para la datación de las placas identificativas.

Novelda, ciudad que nunca se ha dedicado a la producción cerámica, supo y pudo estar a la moda en cuanto a las técnicas decorativas y arquitectónicas se refiere, gracias al auge de una burguesía urbana enriquecida con la actividad comercial. Y muestra de ello es la gran variedad de motivos decorativos de los azulejos que hemos documentado, procedentes de las fábricas de Valencia, a pesar de que muchos fueran originarios de Onda, como se observa en una serie de catálogos donde aparecían los modelos que circulaban por las distintas fábricas azulejeras, algunas de las cuales dejaban en el reverso de los azulejos la impronta con el nombre de la fábrica para dejar constancia de dónde se había elaborado. Sin embargo nosotros no hemos podido comprobar en nuestro caso si los azulejos llevan marca de fábrica puesto que se encuentran colocados en su contexto original.

Por lo que respecta a la azulejería conservada en los interiores de las viviendas, tan solo hemos podido obtener una mínima muestra de lo que seguro supone un conjunto muy rico, pues tenemos constancia de que son numerosas las casas que conservan la azulejería original. Sin embargo no hemos podido acceder a ellas ya que se trata de propiedades privadas.

A pesar de esto, con este estudio hemos querido dejar constancia de un tipo de patrimonio desconocido y poco valorado para conseguir que en un futuro, esperemos que no muy lejano, se tome en consideración tanto por parte de la población como por parte de las responsabilidades políticas, y se inicien las medidas oportunas para protegerlo, divulgarlo y darlo a conocer, ampliando la colección del Museo Municipal de Novelda con muestras de los distintos azulejos conservados. También esperamos poder completar la investigación con la incorporación de las muestras de azulejería que se encuentran en propiedades privadas. 


\section{BIBLIOGRAFÍA}

Aldeguer Jover, F. (2001). Noveldenses con huella, Novelda.

Aranegui Gasco, C. (1987). Historia de la cerámica valenciana, Valencia.

Benito Goerlich, D. y Jarque, F. (1992). Arquitectura modernista valenciana, Valencia.

Blasco García, C. y Navarro Belmonte, C. (1991). El nucli urbà de Novelda i la seua evolució (de l'expulsió dels moriscos a mitjan segle XIX). Betania, 1991, pp. 21-28. Novelda.

Coll Conesa, J. (2000). Les volades ceràmiques pintades ornamentals al País Valencia. Aproximació al cas de Castelló, en Llobregat Conesa Scripta in honorem II, pp. 423-451. Alicante.

Escolano Gómez, F. (1959). Así fue Novelda. Betania 1959, s.p. Novelda.

Escolano Gómez, F. (1960). Novelda en el último cuarto del siglo XIX. Betania 1960, s.p. Novelda.

Estall i Poles, V.J. (2000). Catálogo de la colección de azulejos de serie del siglo XIX. Museo del Azulejo. Onda, Castellón.

Estall i Poles, V.J. (1997). La industria cerámica en onda. Las fábricas, 1778-1997, Onda.

Feliu Franch, J. (1998). La cerámica arquitectónica de Onda en el siglo XIX. Tutor: Víctor Manuel Mínguez Cornelles. Universitat Jaume I (Castellón). Tesis, Historia del Arte, Diciembre 1998.

Ferre Morales, A. (2007). La cerámica arquitectónica. Su conservación y restauración. Sevilla.

García Antón, I. (1997). El arte modernista en Novelda. Novelda.

García Antón, I. (1993). Los intérpretes del arte modernista en Novelda. Actas del VIII Congreso Español de Historia del Arte, (Cáceres 1990) pp. 467-472.

García Antón, I. (1993). Nuevas aportaciones artísticas sobre el Santuario de la Magdalena de Novelda. Primer Congreso de Historia del Arte Valenciano, (Valencia 1992) pp. 501-505.
Gil Navarro, J.D. (1996). Análisis históricoconstructivo del Santuario de la Magdalena en Novelda. Tutores: Yolanda Spairani Berrio y Luis Ferré de Merlo. Universidad de Alicante. Proyecto Fin de Carrera, Arquitectura técnica (Escuela Politécnica Superior de Alicante).

Gómez Cantó, M.A.; Marhuenda Moltó, J. (1999). Las caras que nos contemplan. Novelda.

González Hernández, M. A. (1996): Novelda, cronología de su historia. Betania, 1996 pp. 49. Novelda.

Hardy, W. (1997): Guía del Art Nouveau Estilo. Madrid.

Lang, G. (2004). 1000 Azulejos. 2000 años de cerámica decorativa. España.

Martínez Martínez, J.J. (1999). Estudio histórico-artístico sobre los edificios del siglo XIX y principios del XX de Novelda. Tutores: Miguel Louis Cereceda y Yolanda Spairani Berrio. Universidad de Alicante. Proyecto Fin de Carrera, Arquitectura técnica (Escuela Politécnica Superior de Alicante), Junio 1999.

Martínez Martínez, J.J. (2001): La transformación urbana y arquitectónica de Novelda en el cambio de siglo. VV. AA. Novelda de Villa a Ciudad 1901, pp. 51-60. Novelda.

Navarro Poveda, C. (1994). Retablos cerámicos de Santa María Magdalena localizados en Novelda. Revista la Santa, no 8, pp. 22-28. Novelda.

Navarro Poveda, C. (2002). Los aleros pintados. Elementos ornamentales en la arquitectura popular en Novelda. Betania 2002, pp. 93-95. Novelda.

Pacheco Sánchez, T. (1997). Cerámica española. Summa Artis. Historia general del arte, XLII, pp. 487-512. Madrid.

Payá Abad, C. (2007). Función, técnica y forma. Un recorrido por el Centro Cultural GómezTortosa: Análisis descriptivo. VV. AA. Modernismo en Novelda. El centro Cultural Gómez- Tortosa, pp. 39-103. Novelda. 
Pérez Guillén, I. (1998). Cerámica arquitectónica valenciana: los azulejos de serie (s. XVI- XVIII) Tomo I y II. Oliva.

PérezGuillén,I.(2000).Cerámicaarquitectónica. Azulejos valencianos de serie. El siglo XIX, Tomo I, II y III. Badalona.

Garín, F.V., Soler, M.P., Domínguez, E. (1980). Cerámica valenciana: segles XIII a XIX, Catálogo de la exposición. Colección Cultura Universitaria Popular, Valencia.

VV. AA. (1990). Conjunto, casas y elementos de interés histórico- artístico del núcleo antiguo de Novelda. Cuadernos de la Mola. Creación e investigación, Marzo 1990 no 2, pp. 5-18. Novelda.

VV. AA. (1990). Conjunto, casas y elementos de interés histórico- artístico del núcleo antiguo de Novelda. Cuadernos de la Mola. Creación e investigación, Mayo 1990 nº 3, pp. 3-21. Novelda.

VV. AA. (1991). Conjunto, casas y elementos de interés histórico- artístico del núcleo antiguo de Novelda. Cuadernos de la Mola. Creación e investigación, Febrero 1991 no 5, pp. 3-20. Novelda.

VV. AA. (1994). La casa museo modernista de Novelda. CAM Alicante.

VV. AA. (2003). Historia, arte y tradición de los azulejos valencianos. Museo de Onda. Onda.

VV. AA. (2006). Donació de la col-lecció de taulells Sergio León. Exposició temporal del 22 de juny al 15 de setembre. Onda.

\section{AMN}

Cabildos: 1833, 1834 1835, 1836, 1837, 1838, 1839, 1880, 1881, 1882, 1883, 1884, 1885 ,1886, 1887, 1888, 1889, 1890, 1891, 1892.

\section{RECURSOS ELECTRÓNICOS}

Coll Conesa, J. (2009). La cerámica valenciana. Apuntes para una síntesis, [en línea]. AVECGREMIO.

http://publicaciones.avec.com/historia.html, [consulta mayo, junio, julio y agosto de 2011]. 